\title{
Review: metformin used alone or combined with clomifene may improve ovulation rates in the polycystic ovary syndrome
}

Lord JM, Flight IH, Norman RJ. Metformin in polycystic ovary syndrome: systematic review and meta-analysis. BMJ 2003;327:951-6.

Lord JM, Flight IH, Norman RJ. Insulin-sensitising drugs (metformin, troglitazone, rosiglitazone, pioglitazone, D-chiro-inositol) for polycystic ovary syndrome. Cochrane Database Syst Rev 2003;(3):CD003053.

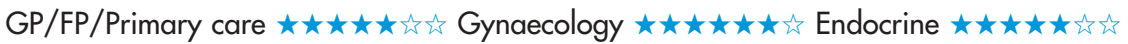

In women with the polycystic ovary syndrome (PCOS), what is the effectiveness of insulin sensitising drugs?

\section{METHODS}

Data sources: the Cochrane Menstrual Disorders and Subfertility
Group trials register (December 2002), the Cochrane Central
Register of Controlled Trials (Cochrane Library, Issue 4, 2002),
Medline (1966 to December 2002), and EMBASE/Excerpta
Medica (1985 to December 2002); bibliographies of relevant
studies; and pharmaceutical companies.
Study selection and assessment: randomised controlled trials
(RCTs) that compared insulin sensitising drugs with placebo, no
treatment, or an ovulation inducting agent in women with PCOS
based on biochemical or ultrasonographic evidence. Studies
were assessed for allocation concealment, blinding, loss to follow
up, non-compliance, and intention to treat analysis.
Outcomes: clinical (including live birth rate, clinical pregnancy
rate, spontaneous ovulation, menstrual cyclicity, and adverse
pregnancy events) and biochemical outcomes.

\section{MAIN RESULTS}

15 RCTs (997 women) were included. 13 RCTs $(n=543)$ used metformin, $1(n=410)$ used troglitazone, and $1(n=44)$ used dchiro-inositol. Blinding was present in all but 1 trial. Women had PCOS only in 3 RCTs, PCOS with obesity in 5 RCTs, PCOS with clomifene resistance in 6 RCTs, and PCOS with clomifene sensitivity in 1 RCT. Women's mean age ranged from 21-32 years. The duration of the trials ranged from 4-44 weeks (median $10 \mathrm{wk}$ ). Metformin improved ovulation rate (table), reduced systolic blood pressure (weighted mean difference [WMD] $-9 \mathrm{~mm} \mathrm{Hg}, 95 \% \mathrm{CI}-15$ to -3 ), and reduced diastolic blood pressure (WMD $-6 \mathrm{~mm} \mathrm{Hg}, \mathrm{CI}-10$ to -2 ) more than placebo or no treatment. Metformin plus clomifene improved ovulation and clinical pregnancy rates more than clomifene alone (table).

For correspondence: Dr J M Lord, Derriford Hospital, Plymouth, UK jonathan_lord@hotmail.com

Source of funding: Reproductive Medicine Unit, University of Adelaide.

\section{CONCLUSION}

Metformin used alone or combined with clomifene is effective for improving ovulation rates in women with the polycystic ovary syndrome.

Abstract and commentary also appear in ACP Journal Club.

\section{Commentary}

DOS is characterized by oligo-ovulation, irregular menses, and symptoms of androgen excess, such as hirsutism and acne. It is a common endocrinopathy affecting 5-10\% of women of reproductive age. Insulin resistance is felt to play a key role in most women with PCOS, therefore insulin sensitising agents have moved to the forefront of management of PCOS. No large scale, long term studies of their use in PCOS have been reported thus far. The meta-analysis by Lord et al combines the results of $15 \mathrm{RCTs}$ using insulin sensitising agents in the treatment of PCOS. The primary outcome measure in 11 of the 15 RCTs was ovulation. Because data are mainly available for metformin use, the conclusions are essentially restricted to this drug.

Support for improvement in ovulation with metformin alone is based on analysis of 7 RCTs. A relative benefit increase exists for metformin compared with placebo in achieving ovulation. The length of the trials is short term, ranging from 4-16 weeks, suggesting that improvement occurs early in treatment. Pregnancy rate is not reported as a primary outcome in the reviewed trials, and the trials did not control for other causes of infertility.

The principal method of ovulation induction for women with PCOS for the past 30 years has been clomiphene citrate. Metformin alone has not been shown to be better than clomiphene in a head to head trial of ovulation induction. Such a trial is currently underway. From this metaanalysis, one cannot conclude at this time that metformin is the first line ovulation induction therapy.

Few included RCTs reported biochemical or metabolic outcomes. Hence, few conclusions can be drawn about these outcomes.

This meta-analysis supports a role for metformin in the restoration of ovulation in PCOS. Long term data on pregnancy rates and changes in metabolic variables are needed. Such factors as lifestyle modification and weight reduction also warrant further study.

Kathleen $M$ Hoeger, MD David S Guzick, MD, PhD University of Rochester School of Medicine and Dentistry Rochester, New York, USA

Metformin $v$ placebo or no treatment and metformin plus clomifene $v$ clomifene alone for the polycystic ovary syndrome*

\begin{tabular}{llll}
\hline & Weighted event rates & & \\
\cline { 2 - 2 } Outcomes at median 10 weeks & Metformin v placebo or no treatment & RBI (95\% CI) & NNT (CI) \\
\hline Ovulation rate (FEM) & $46 \%$ v 24\% & $96 \%(47$ to 161) & 5 (4 to 8) \\
\hline & Metformin + clomifene v clomifene alone & & \\
\hline $\begin{array}{l}\text { Ovulation rate (REM) } \\
\text { Clinical pregnancy rate (FEM) } \dagger\end{array}$ & $76 \%$ v $42 \%$ & $107 \%(19$ to 260$)$ & 4 (2 to 17) \\
\hline
\end{tabular}

*FEM = fixed effects model; REM = random effects model. Other abbreviations defined in glossary; weighted event rates, $\mathrm{RBI}, \mathrm{NNT}$, and $\mathrm{Cl}$ calculated from data in article.

†Secondary outcome measure in all trials. 